Résumés des conférences et travaux

\title{
Épigraphie grecque et géographie historique du monde hellénique
}

Denis Rousset

\section{OpenEdition}

\section{Journals}

Édition électronique

URL : https://journals.openedition.org/ashp/4219

DOI : $10.4000 /$ ashp.4219

ISSN : 1969-6310

Éditeur

Publications de l'École Pratique des Hautes Études

Édition imprimée

Date de publication : 1 septembre 2021

Pagination : 134-141

ISSN : 0766-0677

\section{Référence électronique}

Denis Rousset, «Épigraphie grecque et géographie historique du monde hellénique », Annuaire de I'École pratique des hautes études (EPHE), Section des sciences historiques et philologiques [En ligne], 152 | 2021, mis en ligne le 14 juin 2021, consulté le 18 juin 2022. URL : http://journals.openedition.org/ ashp/4219; DOI : https://doi.org/10.4000/ashp.4219 


\title{
ÉPIGRAPHIE GRECQUE ET GÉOGRAPHIE HISTORIQUE DU MONDE HELLÉNIQUE
}

\author{
Directeur d'études : M. Denis RousseT
}

Programme de l'année 2019-2020: I. Nouvelles inscriptions d'Asie Mineure: Téos. II. Anciennes et nouvelles inscriptions de Grèce centrale.

\section{Nouvelles inscriptions d'Asie Mineure : Téos}

Prenant la suite des années de séminaire où l'on avait étudié Colophon (20132014), Priène (2014-2015) et Milet (2015-2016, 2016-2017), on a consacré une grande partie de cette année à une autre des cités d'Ionie, Téos (déjà abordée en 2018), cité d'autant plus importante que les recherches topographiques et épigraphiques menées depuis 2010 renouvellent considérablement la documentation. Après avoir dressé le tableau de l'histoire de Téos depuis l'époque archaïque, on a exposé les questions géographiques et présenté la topographie et la cartographie du territoire et de sa ville.

On a analysé les recherches de M. Adak et M. Kadioğlu publiées dans « Die Steinbrüche von Teos und "Marmor Luculleum" ", Philia, 3 (2017), p. 1-43; cf. aussi M. Kadıoglu, « Der Gebrauch von Marmorsorten in der Architektur von Nysa und Teos ", dans T. Ismaelli, G. Scardozzi (éd.), Ancient Quarries and Building Sites in Asia Minor. Research on Hierapolis in Phrygia and other cities in south-western Anatolia: archaeology, archaeometry, conservation (2016), p. 711-723. Dans le riche article de 2017, les auteurs éditent entre autres une dédicace latine à Vénus Pontia, datée d'après l'écriture du $\mathrm{I}^{\mathrm{er}} \mathrm{s} . p$. $C$. et mentionnant un affranchi responsable de l'administration des carrières, a lapicidinis luculleis. Cette inscription est importante parce qu'elle confirme l'origine du célèbre marbre « luculléen », appelé aussi par les Modernes "Africano », qui était fort réputé à Rome en raison du goût que lui avait marqué L. Licinius Lucullus, comme nous l'apprend Pline l'Ancien. Son origine est bien Téos, comme cela avait été avancé par quelques commentateurs de l'Histoire naturelle XXXVI 49, passage où cependant cette origine était restée méconnue, discutée, voire contestée en raison des divergences de la tradition manuscrite. Si les éditions de C. Mayhoff (1887) et de J. André (1981) avaient retenu nascitur autem in Melo insula, il faut en définitive éditer Teo, toponyme qui est à préférer à plusieurs leçons ou corrections proposées; il doit en aller de même chez Isidore, Étymologies XVI 5, 17 (Luculleum marmor nascitur in Melo insula, dans l'édition de J. Féans Landera, 2011, avec la discussion ad loc.), auteur qui a puisé chez Pline. Ainsi désormais corrigé et établi comme l'ont mis en évidence M. Adak et M. Kadioğlu, le texte de Pline implique que Téos était bel et bien, malgré les doutes de certains commentateurs, désignée comme une insula, comme il ressort également de sa mention dans $H N \mathrm{~V}, 138$, et ce en dépit de sa situation topographique telle que nous la voyons aujourd'hui. On peut se demander si cette perception d'alors du site de Téos, qui de 
nos jours apparaît comme inexacte, n'est pas due à la position de l'acropole sur un isthme qui était assurément plus mince dans l'Antiquité, entre les deux ports de la ville, au nord et au sud. Ainsi, l'article de M. Adak et M. Kadioğlu démontre combien, entre des passages mal établis des auteurs et une inscription nouvelle, la confrontation est riche de fructueux résultats à la fois pour l'établissement des textes et pour la géographie antique.

On a d'autre part étudié les fortifications de Téos à la lumière des textes (auteurs et inscriptions) et des vestiges archéologiques, en retraçant notamment leur redécouverte depuis les explorations françaises des années 1920 et jusqu'à E. Taşdelen et Y. Polat, Philia, 4 (2018), p. 173-199: «New Investigations, Finds and Discoveries Concerning the Hellenistic City Walls of Teos », article qui présente des progrès importants dans la connaissance de leur tracé. On a examiné également les originales inscriptions gravées en divers points de la fortification, certaines déjà connues anciennement et éditées par F. G. Maier, Gr. Mauerbauinschriften, ${ }^{\text {os }}$ 62-68, d'autres nouvellement publiées en 2018. Parmi ces textes informant du financement des travaux de fortification, on a commenté en particulier les inscriptions Maier $n^{\text {os }} 63$ et 65 , cette dernière rééditée en 2018 d'après la pierre retrouvée. Cette nouvelle édition (Taşdelen-Polat $\mathrm{n}^{\circ} 4$ ) appelle plusieurs remarques : les éditeurs présentent aux 1. 1-2 le nom

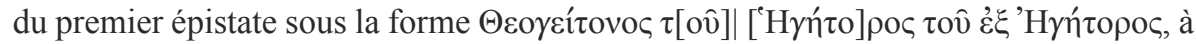

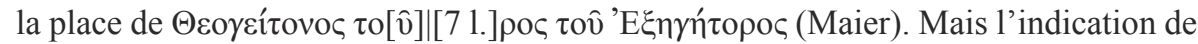
la filiation par $\tau 0 \hat{v} \xi \xi$ paraît bien singulière : faut-il vraiment écarter un anthroponyme, certes hapax, 'E $\xi \eta \eta \dot{\eta} \tau \omega \rho$, qui pourrait d'ailleurs être à la fois celui du grand-père et

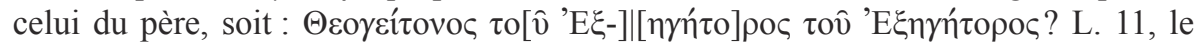
texte indique une dépense de 37 drachmes «d'Alexandre », et non pas 36 (Maier) ni 38 (Taşdelen-Polat). La mention de drachmes à l'effigie d'Alexandre n'implique pas qu'il s'agisse nécessairement (Taşdelen-Polat, p. 183-184) d' «Alexandres » émis par la cité de Téos elle-même, ni donc non plus que cette dépense se place nécessairement à l'époque de pareilles émissions téiennes. En effet, l'essentiel de ces dépenses de fortification, comme le montre toute la série de documents, était payé en drachmes locales, et les drachmes d'Alexandre ne sont que de modestes compléments, qui pourraient justement ne pas correspondre chronologiquement aux émissions par Téos d' «Alexandres » (hypothétiquement destinées à financer de ces travaux); ces pièces pourraient en fait avoir été faits thésaurisées depuis plus ou moins longtemps, et d'autre part avoir été émises par d'autres cités que Téos, suivant le même étalon et le même type, l'un et l'autre largement répandus alors. Notons enfin, pour la chronologie, que E. Taşdelen et Y. Polat ont justement fait remarquer que le texte $\mathrm{n}^{\circ} 65$ est gravé stoichèdon, caractéristique dont on doit tenir compte dans la discussion de sa datation, ainsi que de la chronologie des travaux de fortification. Doit-on en effet admettre que les travaux en divers points de l'enceinte s'échelonnent de la fin du III ${ }^{\mathrm{e}}$ au courant du $\mathrm{II}^{\mathrm{e}} \mathrm{s}$. $a$. $C$. (Taşdelen-Polat, ibid.) et serait-ce par conséquent un cas de gravure stoichèdon assez tardif? Si oui, faudrait-il en rapprocher la fameuse inscription des pirates à Téos, où L. Meier a remarqué l'emploi du stoichèdon (Chiron, 47 [2017], p. 116-117; cf. P. Hamon, Chiron, 48 [2018], p. 371-374, pour la chronologie de cette inscription). On doit également rappeler l'usage sans doute prolongé du stoichèdon dans la voisine Érythrées, e. g. I. Erythrai 34, et d'autre part, à Colophon 
vers 180-160, dans le décret pour le prince attalide Athénaios. Le stoichèdon est-il donc resté en pratique dans cette partie de l'Ionie plus tardivement qu'ailleurs, ne serait-ce qu'à la faveur de l'activité de quelques lapicides locaux?

Quoi qu'il en soit de la chronologie précise de l'usage du stoichèdon, on pourrait être enclin à remonter l'inscription relative aux fortifications Maier $\mathrm{n}^{\circ} 65$ plus ou moins haut dans le $\mathrm{III}^{\mathrm{e}} \mathrm{s}$., ce qui impliquerait que les travaux durèrent fort longtemps, du courant de ce $\mathrm{III}^{\mathrm{e}} \mathrm{s}$. jusque peut-être dans le $\mathrm{II}^{\mathrm{e}} \mathrm{s}$. $a$. $C$. À la question de la limite chronologique inférieure est liée celle de la datation d'un nouveau relevé de dépenses relatives aux fortifications, publié par E. Taşdelen et Y. Polat sous leur $\mathrm{n}^{\circ} 1$, que son écriture pourrait parfaitement assigner à la fin du $\mathrm{III}^{\mathrm{e}} \mathrm{s}$. Pourtant, les éditeurs ont daté

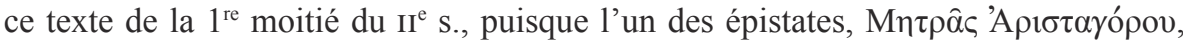
pourrait selon eux n'être autre qu'un des timouques dans les actes de location immobilière publiés par M. Adak et K. Stauner (voir ci-après). Mais la date de ce texte est elle-même discutable et pourrait être descendue dans la $2^{\mathrm{e}}$ moitié du $\mathrm{II}^{\mathrm{e}} \mathrm{s}$., si bien que

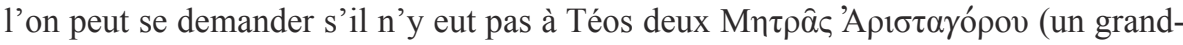
père et son petit-fils, homonyme?). Il en résulterait qu'il n'y aurait plus à dater au $\mathrm{II}^{\mathrm{e}} \mathrm{s}$. le nouveau relevé de dépenses et que les travaux en divers points de l'enceinte pourraient s'être déroulés ou avoir débuté plus ou moins tôt dans le $\mathrm{III}^{\mathrm{e}} \mathrm{s}$., si l'on en juge du moins par les deux textes qui sont à la fois les plus complets et illustrés pour la paléographie (Maier $n^{\circ} 65$ et Taşdelen-Polat $n^{\circ} 1$ ). Ce que montre en outre l'ensemble des inscriptions relatives aux fortifications de Téos maintenant connues, c'est que, au cours des travaux, on a procédé à divers réparations ou remplacements d'assises, en laissant coexister dans certaines sections de la courtine la pierre et la brique,

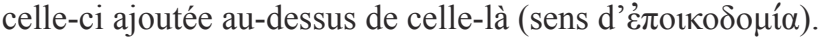

On a consacré plusieurs séances à la superbe inscription intacte $(581$ 1.) publiée avec célérité et compétence par M. Adak et K. Stauner, Philia, 4 (2018), p. 1-25: «Die Neoi and das Temenos des Dionysas. Eine hellenistische Pachturkunde aus Teos »; cf. aussi C. P. Jones, ZPE, 212 (2019), p. 109-114 : « The Lease of a Heroic

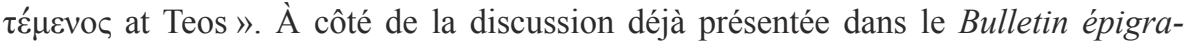
phique 2019, n 419, p. 577-580, ce nouveau joyau de l'épigraphie téienne suscite bien des remarques et questions. On s'est d'une part interrogé sur la date : l'écriture ne pourrait-elle convenir aussi bien à la $2^{\mathrm{e}}$ moitié $d u \mathrm{II}^{\mathrm{e}} \mathrm{s}$. qu'au $2^{\mathrm{e}}$ quart du

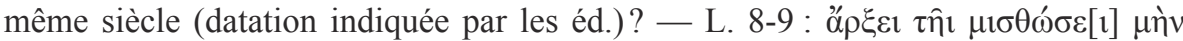

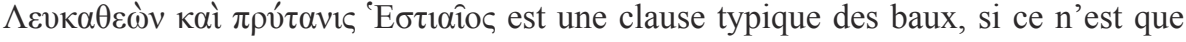
les documents attiques libellent cette indication au temps présent et avec le régime au

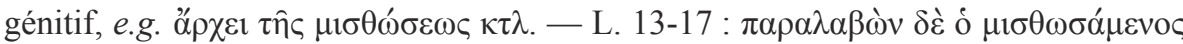

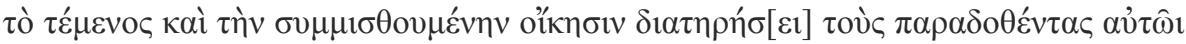

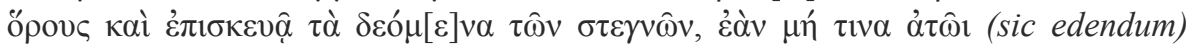

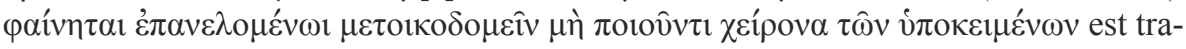
duit par les éd. : « (...) wenn ihm nach der Erlangung (Besitznahme des Pachtobjekts) nicht irgendetwas umzubauen (oder: anderswohin zu bauen) nötig erscheint », et par C. P. Jones « ... seem to him on taking them over to need rebuilding ». Cependant, $\dot{\varepsilon} \pi \alpha v \varepsilon \lambda \mathrm{\rho} \mu \dot{v} v \omega \iota$ ne signifie pas la prise de possession du bien loué (puisque d'ailleurs toutes ces clauses du contrat s'entendent une fois que le locataire aura pris possession, $\pi \alpha \rho \alpha \lambda \alpha \beta \omega v)$. On traduira : «Le locataire réparera ceux des abris qui en ont besoin, à 
l'exception de ceux qu'il lui paraîtra bon, après les avoir démontés, de reconstruire,

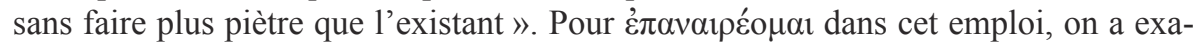
miné le parallèle fourni par I. Délos 290 1. 179, avec le commentaire J.-C. Moretti, $E A D, 42$. Le théâtre I, p. 195-197. Il s'agira donc sans doute de réutiliser les mêmes matériaux pour " reconstruire », voire " reconstruire ailleurs », suivant les deux sens

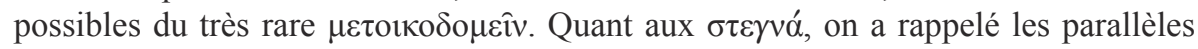

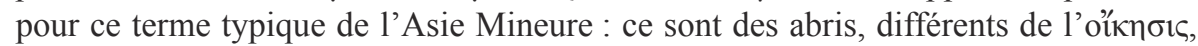
qui sont souvent en relation avce l'exploitation agricole ou artisanale; ils étaient sans doute dans le cas présent situés sur des parcelles attenantes ou du moins liées

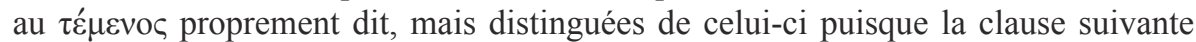

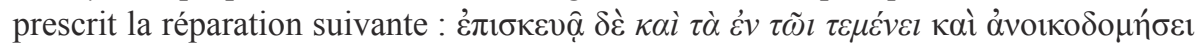

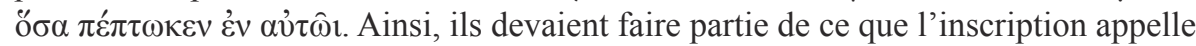

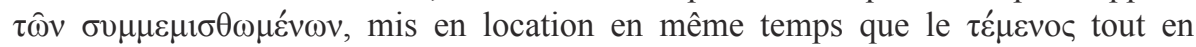
étant cependant distincts (1. 32 et 37), et ils étaient donc sans doute sur des parcelles

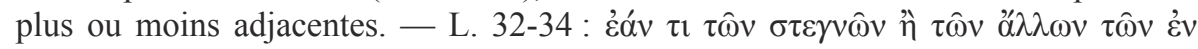

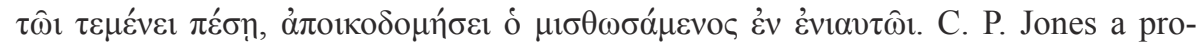

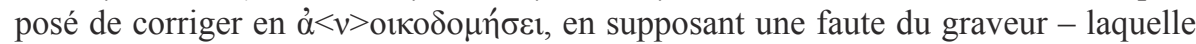
serait presque unique dans ce texte de 58 lignes -, et en alignant cette forme sur

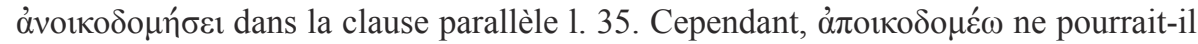
signifier « construire, réparer en comblant, en complétant »? - L. 40, le paiement du héraut est dans ce texte l'indice discret, mais le plus net d'une procédure d'adjudication (voir le parallèle dans un bail de la toute proche Clazomènes, I. Pernin, Baux ruraux $\mathrm{n}^{\circ} 161$ ), laquelle pourrait également ressortir de la clause 1.50 cóv $\tau 1$

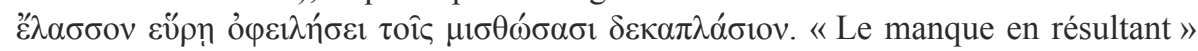

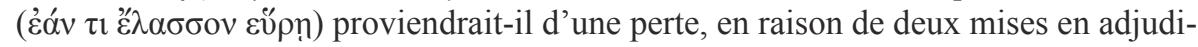
cation successives, entre un plus disant, finalement considéré comme défaillant, et un moins disant lors de secondes enchères? Ou bien serait-ce la perte de loyer provoquée par le report pour le début de la location depuis le jour initialement prévu jusqu'au début effectif de la mise en location, une fois trouvé un nouveau locataire pour rem-

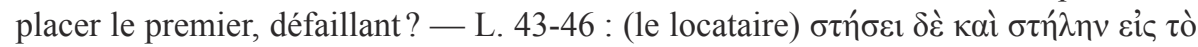

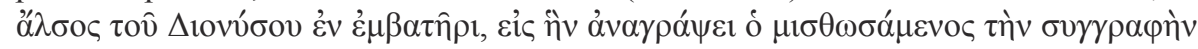

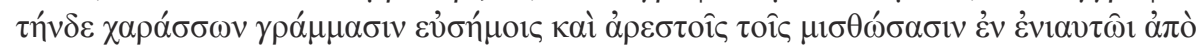

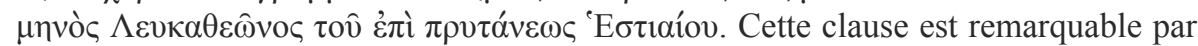
la précision sur les caractères de la gravure, et elle montre en outre que la version gravée sur pierre était vraiment destinée à être lue, et donc sans doute non dénuée elle-même de valeur probante. On a examiné le sens de l'indication $\dot{\varepsilon} v \dot{\varepsilon} \mu \beta \alpha \tau \hat{\imath} \rho$, qui n'est sans doute pas « ein Sockel» (éd.), ni « a mortise» (Jones). En effet, pour les innombrables stèles dont les textes épigraphiques prévoient l'érection, on ne précise pas qu'elles soient érigées dans une base, puisque c'était évidemment le cas le plus courant. Ce que l'on définit au besoin, c'est l'endroit précis d'érection (e. $g$. «dans » l'agora, l'acropole, le gymnase, le temenos, le hiéron, le « lieu le plus en

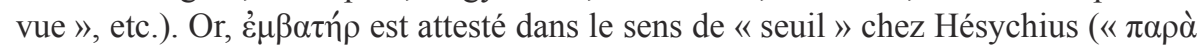

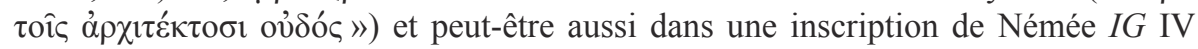
481 (le sens d'embarcadère dans Athenian Agora XIX Leases L 4b 1. 15 est-il bien certain?). On a d'autre part réexaminé les sens de mots parents, í 
$\beta \alpha \tau n ́ \rho$, lequel signifie assurément à la fois base de stèle dans les inscriptions de Délos, mais aussi seuil selon Pollux II 200. D'autre part, on s'est interrogé sur l'emplacement effectif de la stèle à Téos, qui fut trouvée « im Bezirk des Dionysos-Tempels, an dessen Südwestecke auf der Rückseite der Weststoa, nur wenige Meter östlich der hellenistischen Stadtmauer. Sie dürfte ursprünglich innerhalb der Grenzen des Temenos (vgl. Z. 43 : ớ

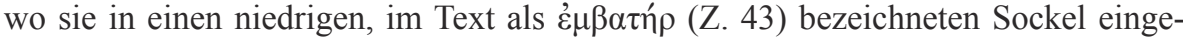
lassen war » (M. Adak et K. Stauner, Philia, 4 [2018], p. 2). La stèle n'était-elle donc pas en réalité fichée dans un seuil ou un emmarchement « vers l'entrée »? Et pourrait-

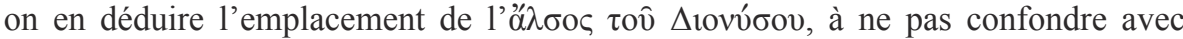
le «Temenos » entourant le temple? Cet $\alpha$ $\alpha \sigma o \varsigma$ se trouvait-il à l'intérieur de l'enceinte urbaine, et donc entre la fortification et le temple, ou bien immédiatement hors les murs, et serait-ce en son entrée que l'on avait érigé la stèle? On doit encore se

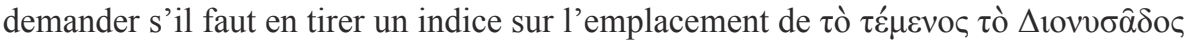

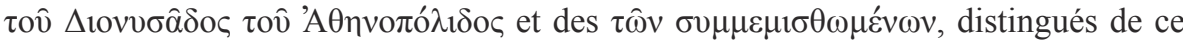
$\tau \varepsilon \dot{\mu} \mu \varepsilon v o \varsigma$, qui sont objets de l'acte de location : étaient-ils donc en tout ou en partie dans la ville ou dans la zone péri-urbaine?

À ces questions est liée celle des lieux et éventuellement constructions utilisés pour le culte annuel en l'honneur du héros : les 1. 23-25 prévoient en effet le déroule-

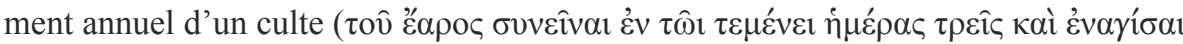

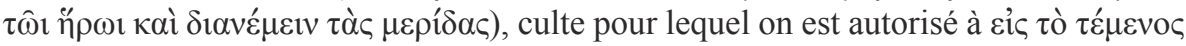

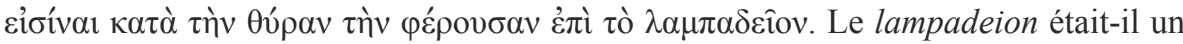
local pour des torches et se trouvait-il dans l'enceinte même du temenos? Et faut-il supposer qu'il existait une course au flambeau en l'honneur du héros? On a d'autre

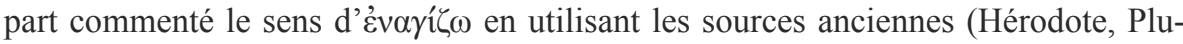
tarque) et les commentateurs récents (Casabona, Ekroth, Pirenne-Delforge) et en soulignant que l'inscription de Téos fournit sans doute pour le verbe un des emplois

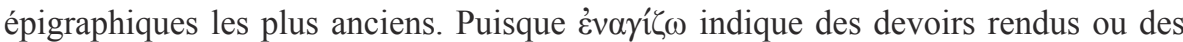
offrandes faites à un défunt, faudrait-il en déduire que la tombe même du héros était dans le temenos mis en location, et, si tel était le cas, le $\tau \varepsilon ́ \mu \varepsilon v o c$ était-il intra muros ou extra muros? Enfin, on a replacé dans l'histoire et le développement des cultes héroïques et post mortem au cours de l'époque hellénistique cette nouvelle attestation du culte pour un héros, qui en l'occurrence n'est autre, selon toute vraisemblance, que le mortel, Dionysas, donnant son nom au temenos.

On a clos l'étude en examinant la composition du texte inscrit et la chronologie des opérations (voir déjà $B E, 2019,419$ ). L'ensemble des opérations, depuis la procédure initiale de mise en location jusqu'à la réalisation effective s'est étendu non pas seulement sous deux prytanes successifs, Théotimos puis Hestiaios, comme l'ont pensé les éditeurs, mais depuis déjà la fin de l'année du prédécesseur, non nommé, de Théotimos (1. 8), tandis que l'année de Théotimos devait initialement être la première de la location (1. 28-29), aux termes de la syngraphè initialement rédigée. Mais un délai ralentit la procédure, si bien que la mise en location effective ne devait avoir lieu que sous l'éponyme suivant, Hestiaios, comme on l'indiqua par des précisions chronologiques qui doivent vraisemblablement avoir été insérées ou modifiées a pos-

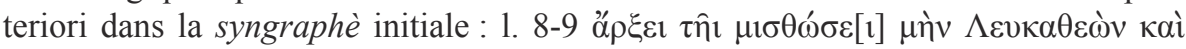




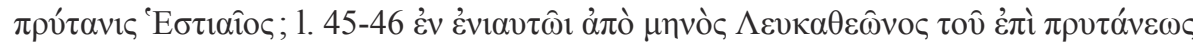

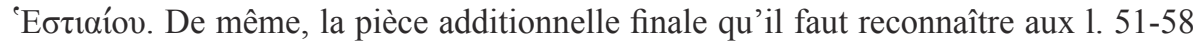
a pour éponyme le même Hestiaios et nomme enfin le locataire désigné et le montant

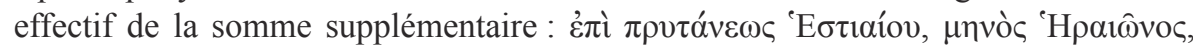

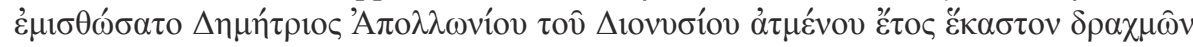

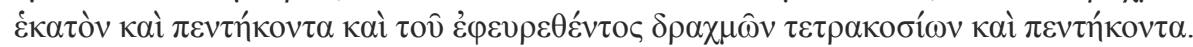

Ainsi, l'analyse chronologique du dossier, composé de trois pièces différentes juxtaposées (1. 1-8; 8-51; 51-58), montre que la mise en location s'est vraisemblablement étendue sur trois années successives. Cela ne fragilise-t-il pas la reconstitution du calendrier de Téos qu'ont proposée les éditeurs? Ceux-ci ont en effet voulu insérer les

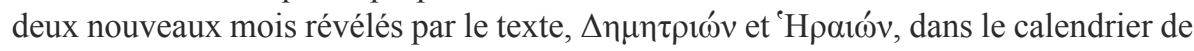
Téos jusqu'ici connu pour cinq mois seulement (cf. L. Meier, Chiron, 2017, p. 158). Or, on ne peut pas actuellement prouver à partir de cette inscription que $\Delta \eta \mu \eta \tau \rho \omega^{\prime} v$ (1. 7) soit le dernier mois de l'année, précédant immédiatement $\Lambda \varepsilon v \kappa \alpha \theta \varepsilon \omega ́ v ~(1.8)$ - lequel est assurément le premier mois de l'année téienne. L'inscription de Téos est en réalité un ensemble composite qui à lui seul ne permet pas d'ordonner de façon sûre et claire les sept mois actuellement connus dans le calendrier téien. - Ajoutons que, depuis l'année 2019-2020, est parue une nouvelle étude de M. Adak et P. Thonemann, « Teos und Abdera in hellenistischer Zeit: Der Jahreskalender, Kulte und neue Inschriften », Philia, 6 (2020), p. 1-34, qui fait connaître un huitième mois, Boédromiôn, et qui reprend l'étude de l'année téienne : voir Bulletin épigraphique 2021.

\section{Anciennes et nouvelles inscriptions de Grèce centrale}

On a proposé à l'étude la convention financière entre Drymos de Phocide et la confédération des Oitaiens, découverte en 1881 et republiée dans $I G$ IX 1, p. 226230, puis dans L. Migeotte, L'emprunt public dans les cités grecques (1984), p. 103$109 \mathrm{n}^{\circ} 29$. Ce texte, gravé sur deux colonnes d'une trentaine de lignes chacune, fut et demeure le premier document épigraphique nous instruisant sur la géographie et les institutions de l'Oitaia, une région voisine de la cité phocidienne de Drymos. Ce texte fut aussi, au moment de sa découverte, l'un des premiers documents nous éclairant sur les rapports entre une cité et un créancier étranger et l'une des rares inscriptions non-delphiques qui touchaient à la chronologie de Delphes et des magistrats phocidiens. Aussi ce document a-t-il été souvent reproduit ou discuté dans les recueils de textes dialectologiques, financiers ou institutionnels, et commenté dans plus d'un article, d'autant plus que l'établissement et la compréhension de ce texte, dans l'ensemble dépourvu de parallèles, faisaient difficulté en plus d'un passage, notamment au début de la col. I et dans la col. II.

Or, de nouvelles possibilités de déchiffrement de la pierre permettent aujourd'hui d'améliorer en plusieurs points l'établissement du texte et d'en proposer un commentaire et une interprétation largement renouvelés, portant sur la succession et le calendrier des opérations financières, sur l'identité des magistrats oitaiens, des témoins et des dépositaires de la convention (notamment le célèbre Étolien Lochagos, connu par les sources écrites pour son exil à Rome et par l'archéologie pour les vestiges de sa maison et de ses archives exhumés à Kallipolis en 1977-1979) et enfin sur le sens du 
terme pylaia dans cette inscription et le rôle qui a été prêté, indûment à notre avis, à l'Amphictionie pylaio-delphique. Après avoir soumis aux auditeurs cette étude, on en a donné la publication dans « L'emprunt de Drymos de Phocide à la confédération des Oitaiens », Journal des savants, 2020, p. 369-428.

D'autre part, on a étudié le dossier des sources relatives à la première guerre mithridatique en Grèce centrale. Après avoir retracé le déroulement des opérations militaires en 86 aux confins de la Béotie et de la Phocide, on a examiné les témoignages de Plutarque (Sylla 17, 19 et 34 et Sur la fortune des Romains 318 D) et Pausanias (IX 40,7) sur les trophées ou monuments commémoratifs de ces opérations, sources que l'on doit confronter à deux découvertes récentes. C'est d'une part le mémorial d'Homoloïchos et Anaxidamos trouvé à l'Ouest de Chéronée sur la colline de Thourion $(B E, 1993,96 ; S E G, 41,448 ; B E, 2009,251 ; 2015,275)$. On a examiné les commentaires qu'a suscités cette découverte aussi passionnante que singulière. Récemment encore, Y. Kalliontzis, dans T. Lucas, C. Müller, A.-C. Oddon-Panissié (ed.), La Béotie de l'archaïsme à l'époque romaine. Frontières, territoires, paysages, Paris, 2019, p. 179-196 : "Le trophée de Sylla à Chéronée », a réétudié le monu-

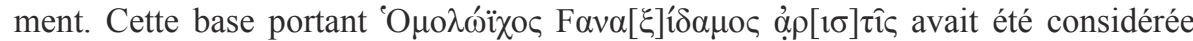
par ses premiers éditeurs $(B E, 1993,96 ; S E G, 41,448)$ comme le monument érigé à la suite de l'intervention militaire au service de Sylla de la part d'Homoloïchos et d'Anaxidamos, désignés comme des «braves » suivant le témoignage explicite de

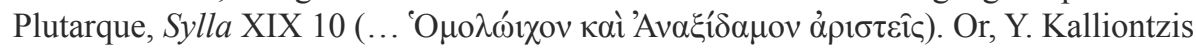
émit des doutes sur sa date, sa forme et sa fonction: il lui paraissait difficile d'admettre que le bloc et sa dédicace aient fait partie d'un trophée gravé et érigé aussi tard que le $\mathrm{I}^{\mathrm{er}}$ s. $a$. C. En 2019, K. a repris l'idée que le dernier mot, loin de désigner des braves

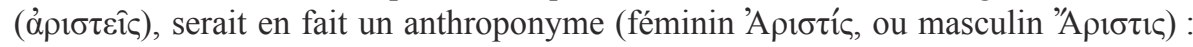
il s'agirait selon K. d'un monument bien antérieur, sans doute pour trois défunts dénommés Omoloïchos, Vanaxidamos et Aristis. Mais comment donc expliquer l'interprétation que transmet de son côté Plutarque? Mécompréhension de sa part, ou détournement volontaire à la faveur d'une « invention des Chéronéens » destinée à montrer leur fidélité à Rome, comme semble le penser K. ? C'est peu vraisemblable : si singuliers que soient ses caractéristiques matérielles, c'est certainement bien à la guerre mithridatique que se rapporte ce monument, qu'il faut d'ailleurs appeler, non pas «trophée de Sylla », mais «mémorial d'Homoloïchos et d'Anaxidamos ». Voir aussi Bulletin épigraphique, 2020, 192.

Enfin, on a étudié ce qui est bel et bien un des trophées que Sylla fit ériger après la bataille d'Orchomène en 86. Découvert entre les localités actuelles d'Orchomène et de Pyrgos, il a donné lieu à une première publication, par E. Kountouri, N. Petrochilos, S. Zoumbaki, dans What's New in Roman Greece? Recent Work on the Greek Mainland and the Islands in the Roman Period, Athènes, 2018, p. 359-368: " The Tropaion of Sulla over Mithridates VI Eupator: A First Approach » (voir $B E$, 2019, 212). C'est de toute évidence le trophée que Plutarque, Sylla XIX 9-10, vit lui-même et dit avoir été érigé en un point où « les troupes d'Archélaos commencèrement à lâcher prise ». D'après la publication, accompagnée d'une mince illustra-

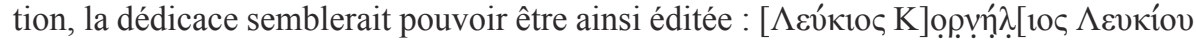

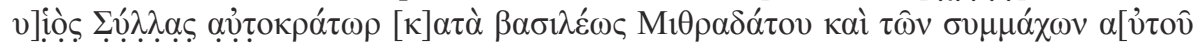




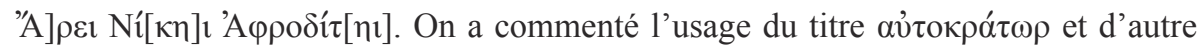
part l'absence, du moins apparente, du surnom 'E $\pi \alpha \varphi \rho o ́ \delta ı \iota \varsigma$, , alors que Plutarque

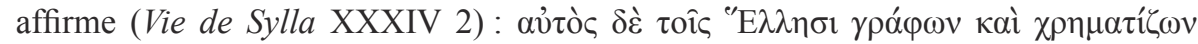

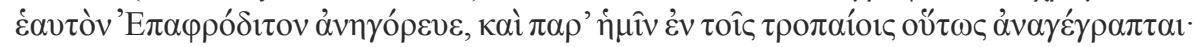

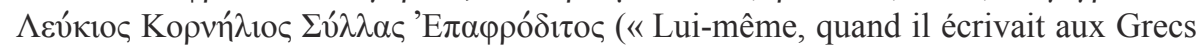
ou répondait à leurs requêtes, se donnait le titre d'"Epaphrodite", et, dans notre pays, son nom est ainsi inscrit sur ses trophées : "Lucius Cornelius Sylla Epaphroditos" ", traduction R. Flacelière, E. Chambry, CUF). Aussi se pose la question suivante : ce

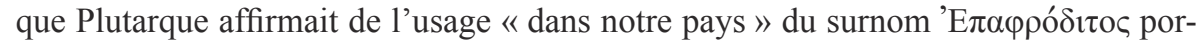
tait-il donc exclusivement sur des trophées érigés dans sa patrie, Chéronée, à la différence de ce trophée qui fut exhumé sur le territoire d'Orchomène, cité pourtant limitrophe?

Ainsi, la découverte de ces monuments inscrits d'Orchomène et de Chéronée donne à la série des sources sur la $1^{\text {re }}$ guerre mithridatique en Grèce centrale une nouvelle profondeur, aussi passionnante que délicate à interpréter. 